\title{
Genomic analyses of unique carbohydrate and phytohormone metabolism in the macroalga Gracilariopsis lemaneiformis (Rhodophyta)
}

Xue Sun ${ }^{1}$, Jun Wu' ${ }^{2}$, Guangce Wang ${ }^{3}$, Yani Kang ${ }^{1,2}$, Hong Sain Ooi ${ }^{4}$, Tingting Shen ${ }^{2}$, Fangjun Wang ${ }^{1}$, Rui Yang ${ }^{1}$, Nianjun $\mathrm{Xu}^{1 *} \mathrm{D}$ and Xiaodong Zhao ${ }^{2^{*}}$

\begin{abstract}
Background: Red algae are economically valuable for food and in industry. However, their genomic information is limited, and the genomic data of only a few species of red algae have been sequenced and deposited recently. In this study, we annotated a draft genome of the macroalga Gracilariopsis lemaneiformis (Gracilariales, Rhodophyta).

Results: The entire $88.98 \mathrm{Mb}$ genome of Gp. lemaneiformis 981 was generated from 13,825 scaffolds ( $\geq 500 \mathrm{bp}$ ) with an N50 length of 30,590 bp, accounting for approximately $91 \%$ of this algal genome. A total of $38.73 \mathrm{Mb}$ of scaffold sequences were repetitive, and 9281 protein-coding genes were predicted. A phylogenomic analysis of 20 genomes revealed the relationship among the Chromalveolata, Rhodophyta, Chlorophyta and higher plants. Homology analysis indicated phylogenetic proximity between Gp. lemaneiformis and Chondrus crispus. The number of enzymes related to the metabolism of carbohydrates, including agar, glycoside hydrolases, glycosyltransferases, was abundant. In addition, signaling pathways associated with phytohormones such as auxin, salicylic acid and jasmonates are reported for the first time for this alga.

Conclusion: We sequenced and analyzed a draft genome of the red alga Gp. lemaneiformis, and revealed its carbohydrate metabolism and phytohormone signaling characteristics. This work will be helpful in research on the functional and comparative genomics of the order Gracilariales and will enrich the genomic information on marine algae.
\end{abstract}

Keywords: Gracilariopsis lemaneiformis, Genomic analysis, Carbohydrate metabolism, Phytohormone signaling

\section{Background}

Red algae (or Rhodophyta) compose an ancient and unique clade of photosynthetic eukaryotes that includes more than 6500 species [1]. Red algae have important economic value as raw materials in the food, medicine and phycocolloid industries. Additionally, red algae have received increasing attention from the scientific community

\footnotetext{
*Correspondence: xunianjun@nbu.edu.cn; xiaodongzhao@sjtu.edu.cn ${ }^{1}$ Key Laboratory of Marine Biotechnology of Zhejiang Province, School of Marine Sciences, Ningbo University, Ningbo 315211, People's Republic of China

${ }^{2}$ School of Biomedical Engineering, Shanghai Center for Systems Biomedicine, Shanghai Jiao Tong University, Shanghai 200240, People's Republic of China

Full list of author information is available at the end of the article
}

due to their genetic contributions to other eukaryotic organisms through secondary endosymbiosis, enabling the tracking of the evolutionary history of many eukaryotic lineages [2]. However, relatively little is known about the genomic and genetic backgrounds of red algae.

The red alga Gracilariopsis lemaneiformis (Gp. lemaneiformis), formerly known as Gracilaria lemaneiformis (G. lemaneiformis) [3], was previously thought to be distributed in China, Japan, Peru, and America. However, Gurgel et al. [4] reported Gp. lemaneiformis was not distributed worldwide and was restricted to the vicinity of Peru. In China, Gp. lemaneiformis has become the third largest cultivated seaweed after Saccharina and Pyropia, and has an annual dry weight of 246 million $\mathrm{kg}$. Wild

(C) The Author(s). 2018 Open Access This article is distributed under the terms of the Creative Commons Attribution 4.0 International License (http://creativecommons.org/licenses/by/4.0/), which permits unrestricted use, distribution, and 
populations of Gp. lemaneiformis are mainly distributed along the northern coast of China, but the high-temperature-tolerant cultivar 981 can be cultivated from the northern to the southern coasts [5]. The cultivated Gp. lemaneiformis are used as a delicious food source for humans, as abalone feed and as a resource for producing agar. In addition, Gp. lemaneiformis is effective at removing nitrogen and phosphorus from seawater and inhibiting red tide causing microalgae [6,7].

The red alga Gp. lemaneiformis contains abundant and diverse carbohydrates such as polysaccharide and agar. The polysaccharides of the algae have antitumor, hypoglycemic and immunomodulatory activities [8-10]. Agar, one of the main carbohydrates in Gp. lemaneiformis, has diverse applications in the food, pharmaceutical, cosmetic, medical and biotechnology industries. Approximately 9600 tons of agar were produced in 2009 (US\$173 million), of which 80\% was obtained from Gracilaria or Gracilariopsis [11]. Although many species of the Gracilariales are good candidates for commercial agar extraction, Gp. lemaneiformis is superior because of its high agar content [12].

Phytohormones, or plant hormones, play a key role in plant growth and development or act as regulators of the defense response against adverse environmental conditions. Phytohormones have similar functions in algae and higher plants [13]. Many phytohormones, including indole-3-acetic acid (IAA), abscisic acid (ABA), jasmonic acid (JA) and salicylic acid (SA), have been detected in Gp. lemaneiformis [14]. However, the phytohormone signaling pathways in algae may be different from those in higher plants.

Up to now, limited information is available regarding the genetic architecture, transcribed genes and metabolic pathways in Gp. lemaneiformis. Although the mitochondrial and chloroplast structures of Gp. lemaneiformis have been investigated $[15,16]$, and although some genomic information is available [17], the limited knowledge regarding this species has impeded genetic studies and cultivation practices. In this study, we report a draft genome of Gp. lemaneiformis using the high-throughput sequencing technology. Then, we discuss the metabolic pathways of agar and other carbohydrates, as well as phytohormone signaling pathways. Our work will offer an invaluable resource for Gp. lemaneiformis and studies on other red algae.

\section{Results}

\section{Genome sequencing and assembly}

In this paper, we assembled a draft genome of $88.98 \mathrm{Mb}$ from tetrasporophytic thalli of Gp. lemaneiformis 981. Approximately 60.7 million paired-end sequencing reads $(2 \times 100 \mathrm{bp})$ were generated using an Illumina HiSeq sequencer. Simultaneously, 623.6 million reads with an average length of $4305 \mathrm{bp}$ were generated using a Pacific Biosciences sequencer. These raw sequencing reads, which represent approximately 189 -fold coverage based on the estimated Gp. lemaneiformis genome size [18], were combined for the genome assembly. Using 62,208 contigs with an N50 of $28,502 \mathrm{bp}, 92.58 \mathrm{Mb}$ of sequences were assembled. A total of 48,035 scaffolds yielded a $94.60 \mathrm{Mb}$ genome with an N50 length of $30,590 \mathrm{bp}$. Ultimately, an $88.98 \mathrm{Mb}$ genome derived from 13,825 scaffolds with lengths equal to or greater than $500 \mathrm{bp}$ was used for further analysis (Table 1). The GC content of the Gp. lemaneiformis genome was $48.13 \%$. The top 20 assembled contigs with the longest and most genes and the corresponding RNA abundance are shown in Fig. 1.

To determine whether the assembled Gp. lemaneiformis genome matched the published data, we mapped the scaffolds to the published mitochondrial genome of $G p$. lemaneiformis [15]. We found that two scaffolds (i.e., scaffold2118 with a length of $13,296 \mathrm{bp}$, and scaffold2119 with a length of $13,237 \mathrm{bp}$ ) fully aligned to the mitochondrial genome, suggesting that the assembly reported here was reliable.

\section{Genome annotation and gene prediction}

Using homology-based and ab initio prediction approaches, we generated a repetitive element dataset, which was used to annotate the Gp. lemaneiformis genome. This investigation led to the identification of $38.73 \mathrm{Mb}$ of repetitive sequences, accounting for $40.94 \%$ of the total assembled algal genome. Except for unclassified repeats, approximately $60.45 \%$ of the repeats were classified into known families (Fig. 2). Class I, long terminal repeat (LTR) retrotransposons, were found to be the most abundant repeat elements $(17.04 \%$ of the entire genome), representing $41.60 \%$ of the total known repeat sequences, including the Gypsy and Copia families. Class II, DNA transposons, were the second most abundant repeat family, accounting for $3.80 \%$ of the genome. In addition to cut-and-paste class II transposable elements (TEs), we identified $1.71 \%$ of rolling-circle transposons ( $\mathrm{RC} /$ Helitron) in this algal genome. Helitrons are transposons that function via a rolling-circle replication

Table 1 Summary of the Gp. lemaneiformis genome assembly

\begin{tabular}{lll}
\hline & Contigs & Scaffolds \\
\hline N50 (bp) & 28,502 & 30,590 \\
Longest (bp) & 525,375 & 525,375 \\
Total number & 62,208 & 48,035 \\
Total size (Mb) & 92.58 & 94.60 \\
Total number ( $\geq 500 \mathrm{bp})$ & - & 13,825 \\
Total size $(\mathrm{Mb})(\geq 500 \mathrm{bp})$ & - & 88.98 \\
\hline
\end{tabular}




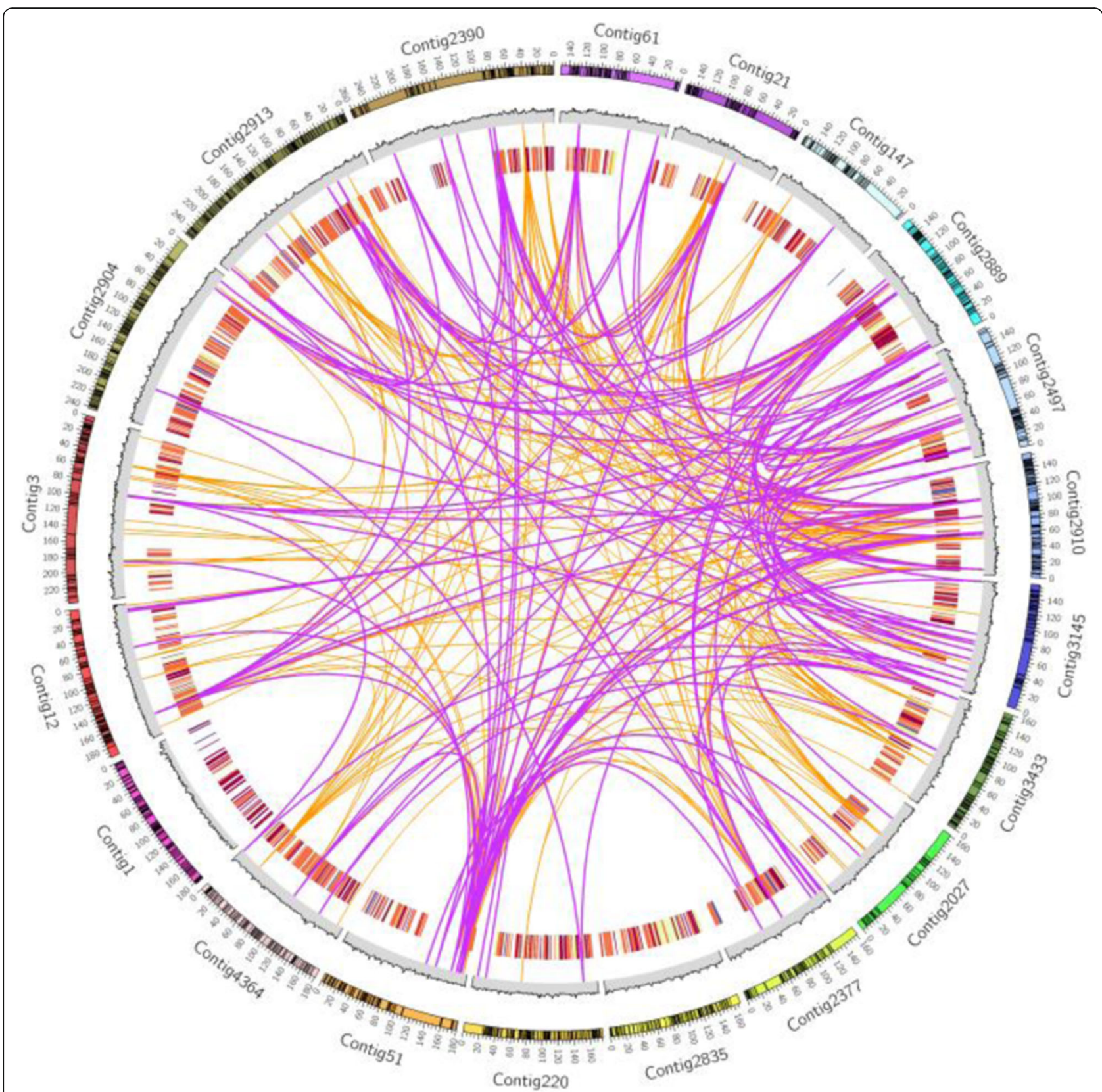

Fig. 1 Circular diagram depicting the genomic features of the top 20 longest contigs. The outer ring represents the top 20 longest contigs, and the black blocks represent the predicted genes. The middle ring shows the GC content of the corresponding contigs in $1 \mathrm{~kb}$ bins. The inner rings show expression of the predicted genes. The blue color indicates low expression, and the red color indicates high expression. The links in the plot represent similarity between the contigs

mechanism and have been reported in $>2 \%$ of Arabidopsis thaliana genomes [19].

To accurately annotate the protein-coding genes in the genome of Gp. lemaneiformis, we used a combined strategy that integrated gene predictions, protein-homolog-based approaches and transcriptome-evidence-based approaches. In total, we predicted 9281 genes with an average length of $1398 \mathrm{bp}$ in the algal genome. Approximately $62.40 \%$ of the predicted coding loci were supported by the RNA-seq data.
The results demonstrate the high accuracy of gene prediction in the Gp. lemaneiformis genome.

\section{Comparative genomics}

A total of 20 genomes from the Chromalveolata, Rhodophyta, Chlorophyta and higher plants were selected for phylogenomic analysis, and the single-copy genes that were shared by these species were used to generate a phylogenetic tree. As shown in Fig. 3a, the 20 species 


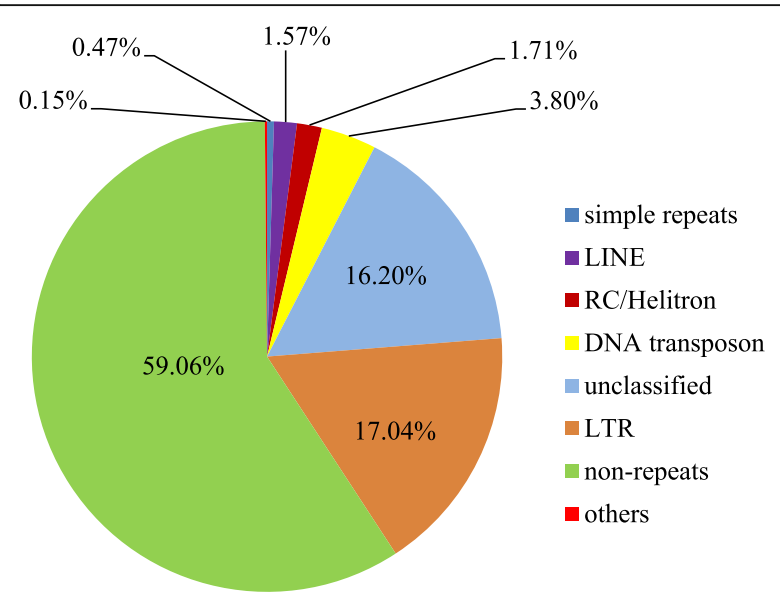

Fig. 2 Repetitive element distribution in the Gp. lemaneiformis genome. LINEs, long interspersed repeated DNA elements. LTR, long terminal repeat; $\mathrm{RC} /$ Helitron, rolling-circle transposon

were divided into the following two main groups in the tree: Archaeplastida and Chromalveolata. The Archaeplastida contained red algae, green algae and higher plants. According to the phylogenetic tree, Gp. lemaneiformis is phylogenetically close to C. crispus, which is a multicellular red alga with a fully sequenced genome.

To investigate the features of the Gp. lemaneiformis genome, we examined the genes that were homologous to those of three other red algae. Of the four species, the marine macroalgae Gp. lemaneiformis and C. crispus belonged to the Florideophyceae; however, Cyanidioschyzon merolae and Galdieria sulphuraria are closely related unicellular thermo-acidophilic red algae (Bangiophyceae and Cyanidiaceae). In total, 6177, 4059, and 4471 common genes were identified after comparing Gp. lemaneiformis with C. crispus, C. merolae, and G. sulphuraria, respectively. Overall, 3502 genes were common across these 4 species (Fig. $3 \mathrm{~b}$ ). The results of this analysis confirmed the phylogenetic proximity between $G p$. lemaneiformis and C. crispus and revealed the broader evolutionarily relationship between macroalgae and unicellular red algae.

In addition, we compared the predicted gene models in the non-redundant protein database of algae and plants using the best-hit method. This analysis yielded top hits from various organisms, and $C$. crispus, G. sulphuraria and A. thaliana were the most frequent species (Fig. 3c).

\section{Gene ontology (GO) and Kyoto encyclopedia of genes and genomes (KEGG) analyses}

To investigate the genes and pathways that are specifically involved in this alga, we performed GO and KEEG analyses using the gene models predicted in this study. Based on the three categories biological process (BP), cellular component (CC), and molecular function (MF), we found that the most frequent GO terms included "oxidation-reduction process" (BP), "ATP binding" (MF), "metabolic process" (BP), "membrane" (CC), "oxidoreductase activity" (MF), "transferase activity" (MF) and "cytoplasm" (CC) (Fig. 4a). In the KEGG analysis, the pathways "biosynthesis of amino acids", "carbon metabolism", and "oxidative phosphorylation" were enriched, containing 104, 93 and 60 genes in the Gp. lemaneiformis genome, respectively (Fig. 4b).

\section{Carbohydrate metabolism analysis Agar biosynthesis-related enzymes}

Based on the agar biosynthetic pathway in red algae [20, 21 ], we compared the homologous genes involved in agar precursor biosynthesis in Gp. lemaneiformis with those in other algae, including the red alga $C$. merolae, the green alga Chlamydomonas reinhardtii, the stramenopile alga Nannochloropsis gaditana, the brown alga Ectocarpus siliculosus, and the diatom Phaeodactylum tricornutum (Fig. 5 and Additional file 1: Table S1). Among these six species of algae, the enzymes phosphomannomutase (7) and GTP-mannose-1-phosphate guanylyltransferase (8) were the most abundant in Gp. lemaneiformis, and phosphoglucomutase (2) was also highly enriched, to the same degree as in C. merolae or P. tricornutum. The numbers of three enzymes (4, 9 and 12) in Gp. lemaneiformis were equal to those in several species. However, the degree of enrichment of phosphoglucose isomerase (alternatively known as glucose-6-phosphate isomerase, 1), UTP-glucose-1-phosphate uridylyltransferase (also known as UDP-glucose pyrophosphorylase, 3) and phosphomannose isomerase (alternatively mannose-6-phosphate isomerase, 6) in Gp. lemaneiformis was second to their enrichment in P. tricornutum (1), C. reinhardtii (3) and $E$. siliculosus (6), respectively. Although enzymes 5, 10 and 11 were not detected, Gp. lemaneiformis had an expanded repertoire of agar biosynthesis-related enzymes.

After the agar precursor is synthesized, a set of genes, including methyltransferases (MTs), sulfotransferases (STs), sulfurylases, pyruvyl transferases (PTs), sulfatases and sulfohydrolases (SHs), are needed to convert the agar precursor to agar [21].

Sulfotransferases are responsible for catalyzing the transfer of a sulfur group from a donor to an acceptor alcohol or amine molecule [22]. In Chondrus, 12 ST-encoding genes have been identified [23]; however, we found eight STs in Gp. lemaneiformis (Additional file 2: Table S2). Of the eight ST-encoding genes, three contigs (Contig145.10, 219.13 and 4885.26) encode carbohydrate sulfotransferases. The carbohydrate sulfotransferases in red algae participate in the synthesis of sulfated polysaccharides. 


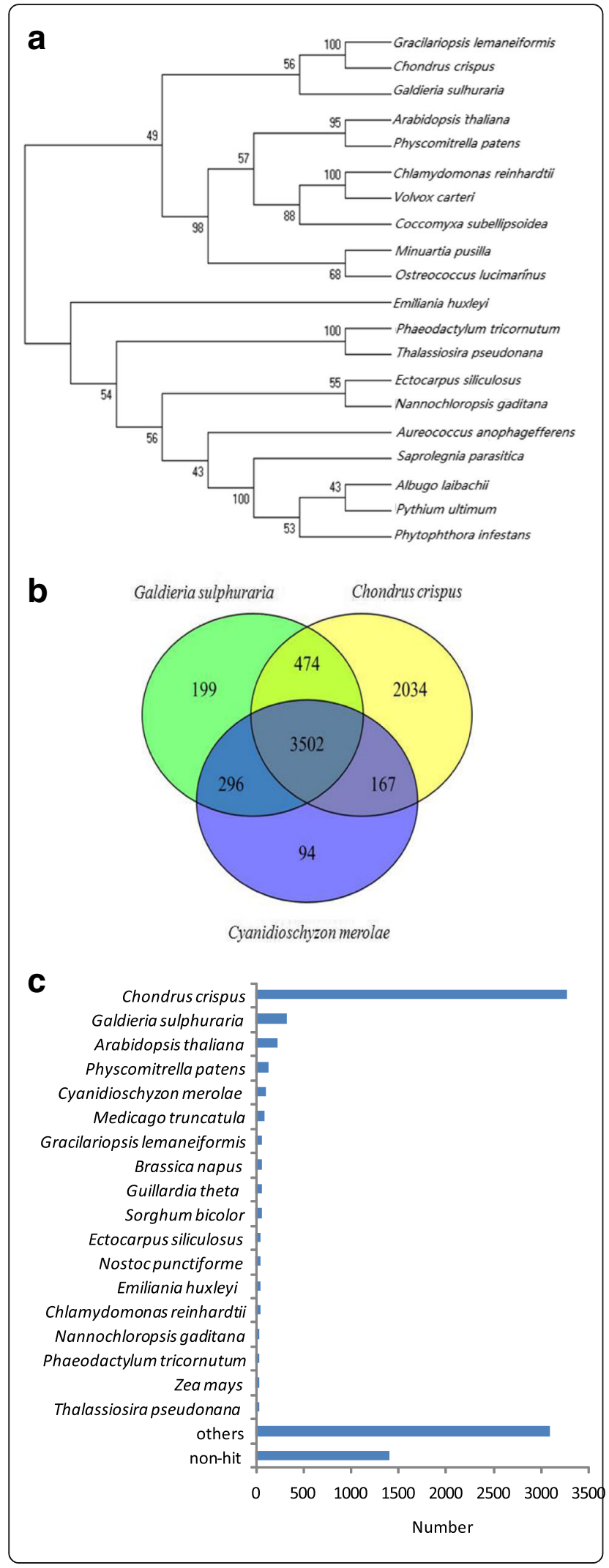

Fig. 3 Comparative genomic analysis of Gp. lemaneiformis. a The phylogenetic tree was generated using the maximum likelihood method based on single-copy genes shared between algal and plant genomes. $\mathbf{b}$ The Venn diagram represents the Gp. lemaneiformis genes shared in the C. crispus, C. merolae and G. sulphuraria genomes. c Gene models of Gp. lemaneiformis are compared with the characterized genomes in the non-redundant protein database using BLASTp. The number of organisms with the top BLASTp hits against Gp. lemaneiformis is indicated

In total, six genes are related to sulfurylases in $G p$. lemaneiformis (Additional file 2: Table S2). Of these genes, four contigs (Contig1123.1, 137.1, 142.3 and 9907.5) belonged to the galactose-2, 6-sulfurylase I family, and two (Contig304.1 and 304.2) belonged to the galactose-2, 6-sulfurylase II family. Galactose-2, 6-sulfurylase specifically transfers aryl or alkyl groups to produce 3, 6-anhydrogalactose residues, which may play a key role in the conversion of the agar precursor to a mature polysaccharide in red algae. In Chondrus, 11 related sulfurylases have been identified, and eight were classified as type-II sulfurylases [23].

Sulfatases are necessary for sulfation modification in the cell walls of algae. Unexpectedly, no sulfatases were found in C. crispus, while nine genes encoding family 1 sulfatases were present in the brown alga E. siliculosus [24]. In the Gp. lemaneiformis genome, we found five sulfatase-encoding genes (Table S2). Of them, one (Contig5126.2) was an alkyl SULF, three contigs (Contig14346.1, 14,346.2 and 14,346.6) included arylsulfatases, and one (Contig14346.3) encoded $\mathrm{N}$-acetylgalactosamine-6-sulfate sulfatase. Therefore, there are different modifications between agar and carrageenans, while some modifications are similar between agar and alginate.

\section{Glycoside hydrolases (GHs)- and glycosyltransferases (GTs)-related enzymes}

In total, $51 \mathrm{GH}$ and $105 \mathrm{GT}$ genes were identified in Gp. lemaneiformis (Additional files 3 and 4: Tables S3 and S4), which were fewer than those identified in the brown alga Saccharina japonica $(130,82)$ [25] but more than those identified in another red alga, C. crispus $(65,31)$ [23]. Seven GH families (i.e., GH19, GH25, GH28, GH42, GH43, GH53 and GH113) and three GT families (i.e., GT11, GT32 and GT61) were found only in Gp. lemaneiformis. Of these families, the GT61 family (11) consisted of 11 genes, while the other nine families each had only one copy. In contrast, several GH and GT families had different members in brown algae and red algae. For example, five GH families (i.e., GH10, GH17, GH30, GH88 and GH114) occurred only in S. japonica, and even more surprisingly, the GH81 family included 53 genes in $S$. japonica but only 1 and 0 in Gp. 

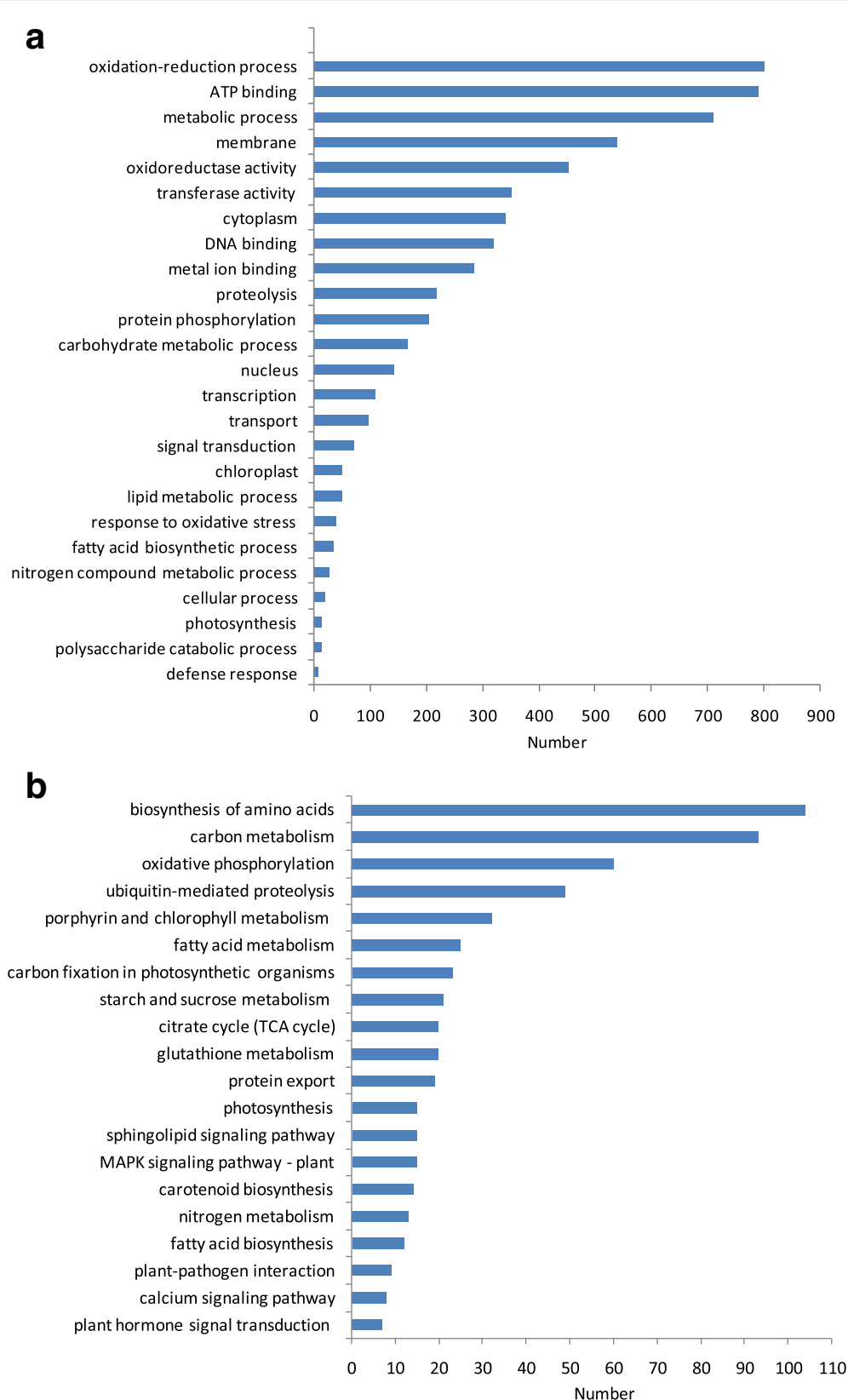

Fig. 4 GO and KEGG analyses of the Gp. lemaneiformis genome. a GO terms derived from the Gp. lemaneiformis gene models. b KEGG pathway lists for Gp. lemaneiformis

lemaneiformis and C. crispus, respectively. In addition, seven GT families (i.e., GT10, GT23, GT48, GT50, GT60, GT68 and GT74) were only present in S. japonica, and family GT23 consisted of 17 genes.

Trehalose, a kind of non-reducing disaccharide, is present in a wide variety of organisms including algae. The metabolic enzymes involved in trehalose include trehalase (family GH37), trehalose-phosphate synthase (TPS, family GT20), trehalose phosphatase and trehalose synthase. The first three trehalose-related enzymes were found in the Gp. lemaneiformis genome (Additional file
5: Table S5). In addition to one trehalose phosphatase gene, three trehalase and four TPS genes were detected in this alga, which are similar to those in C. crispus [23].

Cellulose is an important component of the cell walls of plants and algae. In total, 12 GT2 genes were identified in Gp. lemaneiformis, which was more than the number identified in C. crispus (3) and only half the number identified in S. japonica (24) (Additional file 3: Table S3). Of these genes, five GT2 genes belonged to the cellulose synthase superfamily, which has been classified into nine cellulose synthase-like (CSL) families and 


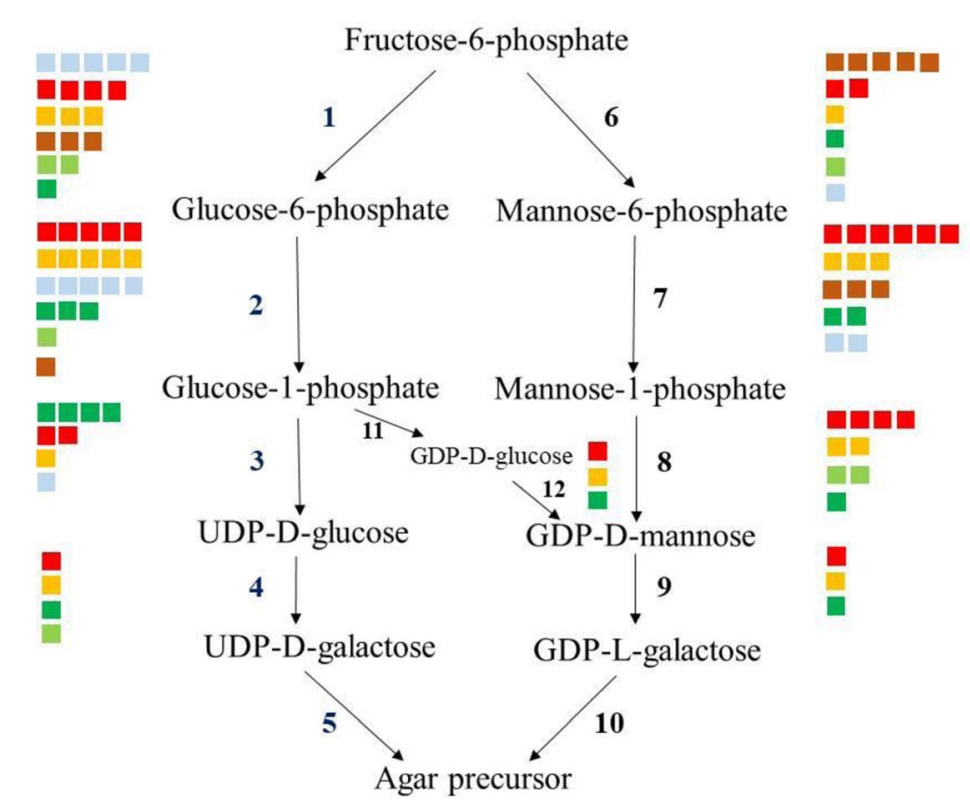

Fig. 5 Analysis of genes potentially involved in agar biosynthesis. Gene homologs in the agar biosynthetic pathways in Gp. lemaneiformis compared with those in C. merolae, N. gaditana, E. siliculosus, C. reinhardtii and P. tricornutum. The colored squares denote the number of homologous genes in each species. The step numbers and their representative enzymes are 1. phosphoglucose isomerase; 2 . phosphoglucomutase; 3. UTPglucose-1-phosphate uridylyltransferase; 4. galactose-1-phosphate uridylyltransferase; $\mathbf{5}$. UDP galactosyltransferase; $\mathbf{6}$. phosphomannose isomerase; 7. phosphomannomutase; 8. GTP-mannose-1-phosphate guanylyltransferase; 9. GDP-mannose-3,5-epimerase; $\mathbf{1 0}$. GDP galactosyltransferase; $\mathbf{1 1 .}$ UDP-glucose pyrophosphorylase; 12. GDP-mannose-3,5-epimerase (the same as 9 )

one cellulose synthase (CESA) family [26]. In addition to the three CESAs identified in the Chondrus genome, another CESA in Rhodophyta has been reported in Porphyra yezoensis and Griffithsia monilis [27, 28]. We identified five CESA or CSL genes in Gp. lemaneiformis. Similar to the CESAs in Chondrus, four CESAs were found in Gp. lemaneiformis (Contig33.5 was excluded because the amino acid sequence it encoded was short), and they were divided into two categories (Additional file 6: Figure S1). One class included CESAs from certain species of red algae, such as Chondrus, Griffithsia, Porphyra, and Pyropia, as well as two contigs (Contigs60.7 and 60.8) from Gp. lemaneiformis. Two other genes (Contig33.6 and 1420.1) clustered with the CESAs from prokaryotic organisms, such as Nostoc and Bacillus, and eukaryotic organisms from Chondrus and Nannochloropsis. Therefore, the plant CSLA and CSLC genes have different origins [29].

\section{Phytohormone signaling analysis Auxins}

IAA and indole-3-acetamide were identified as the main auxins in 11 red algae from the Brazilian coast [30]. In this work, multiple auxin-related genes were found, including auxin transport, auxin efflux carrier and auxin response factor genes (Additional file 7: Table S6). Of these genes, the indole-3-glycerol-phosphate synthases (4) were the most abundant. Moreover, the auxin transport protein BIG (2), auxin efflux carriers (2), and phenylacetic acid degradation proteins (2) were detected in Gp. lemaneiformis.

\section{Abscisic acid}

ABA-related components were also very enriched in this alga. Four 3'(2'), 5'-bisphosphate nucleotidases, which are components of the ABA-activated signaling pathway, were detected (Additional file 8: Table S7). Moreover, ABA-insensitive 5-like proteins (a total of 6) were found in the Gp. lemaneiformis genome. In addition to the key enzymes in ABA synthesis, such as 9-cis-epoxycarotenoid dioxygenase, one farnesylcysteine lyase, which is a negative regulator of ABA signaling [31], was also identified in Gp. lemaneiformis.

\section{Salicylic acid}

SA is a phytohormone that is involved in biotic and abiotic stress. Two SA synthesis pathways have been proposed in plants, including a pathway from cinnamate via the activity of phenylalanine ammonia lyase (PAL) and another pathway from isochorismate via two reactions catalyzed by isochorismate synthase (ICS) and isochorismate pyruvate lyase (IPL) in bacteria. Arabidopsis contains two ICS genes but no IPL genes [32]. Similar to Arabidopsis, one ICS gene but no IPL genes were 
detected in Gp. lemaneiformis. Additionally, as in C. crispus, genes encoding PAL and salicylate synthase were absent [23]. Moreover, one salicylate 1-monooxygenase and one chorismate mutase were detected in Gp. lemaneiformis (Additional file 9: Table S8).

\section{Jasmonates}

JAs, which include various lipid-derived signaling compounds, also play key roles in the growth and development of plants, as well as their stress and defense responses. A series of enzymes, including lipoxygenase (LOX), allene oxide synthase (AOS), allene oxide cyclase (AOC), and 12-oxophytodienoate reductase 3 (OPR3), participate in JA biosynthesis [33]. In higher plants, many enzymes involved in JA synthesis (e.g., six LOXs and four AOCs in A. thaliana) have been characterized $[34,35]$. However, only two LOX genes, not AOS or AOC genes, were observed in the seaweed C. crispus [23]. Similar to C. crispus, two genes encoding LOX were detected in Gp. lemaneiformis (Additional file 10: Table S9). A pairwise comparison showed that the two LOX genes in this alga shared only $17.66 \%$ identity, which was similar to the $20 \%$ identity between the two LOX genes in C. crispus. The two LOX genes in Gp. lemaneiformis were separately distributed among the 14 LOX genes from 10 species in the phylogenetic tree (Additional file 11: Figure S2). Thus, the JAs in red algae might be synthesized through pathways unlike those in higher plants.

\section{Discussion}

Currently, next-generation sequencing techniques, such as the Illumina and 454 platforms, are the main methods used to generate draft genomes. Using the RNA-seq technique, thegenomes of several algae such as $S$. japonica [25], Porphyridium purpureum [36], Nannochloropsis gaditana [37] have been constructed and elucidated. In 2013, a $97 \mathrm{Mb}$ genome was reported, and 3490 genes were identified in the female gametophyte of the wild Gp. lemaneiformis [17]. Here, we present a draft genome of the tetrasporophyte of cultivated Gp. lemaneiformis strain 981 by combining RNA-seq and paired-end-tag (PET) approaches. Based on the size of the published wild Gp. lemaneiformis genome [17], approximately 91.73\% of this algal genome was assembled in this study. The considerable number of repetitive elements is a major structural feature of plant genomes. For example, $73 \%$ of the genome of the macroalga Chondrus crispus (Rhodophyta) consists of repeated sequences [23]. This study indicated approximately $40.94 \%(38.73 \mathrm{Mb})$ repetitive elements in the cultivated Gp. lemaneiformis, however, 54.64\% (44.35 Mb) TEs were revealed in the wild Gp. lemaneiformis [17]. But the GC contents in the two Gp. lemaneiformis genomes were identical ( 48\%) [17].
Agar is one of the most important polysaccharides in the cell walls of red algae, including in the genera Gracilariopsis, Gracilaria and Gelidium. An understanding of the agar biosynthetic enzymes in Gp. lemaneiformis is very important for improving its agar content for the seaweed industry. Although a pathway for agar biosynthesis in red algae has been proposed, it has not been fully elucidated [20, 21]. Several genes encoding galactose-1-phosphate uridylyltransferase, UDP-glucose pyrophosphorylase and phosphoglucomutase have been shown to be related to agar yield in Gracilaria and Gracilariopsis species [38-40]. As compared to green algae and brown algae, in red algae, the enzymes participating in agar biosynthesis are extremely abundant, and the agar synthesis-related genes in Gp. lemaneiformis were more enriched than those in the carrageenan red alga, Chondrus. Up to now, the metabolism of agar remains unclear. For example, the bypass from glucose-1-phosphate to GDP-D-mannose is unconvincing [20, 21]. In addition, Chang et al. [20] reported that UDP galactosyltransferase (5) and GDP galactosyltransferase (10) catalyzed the synthesis of the agar precursor, however, Lee et al. [21] recorded both of them as glycosyltransferase. In contrast to that in higher plants such as Arabidopsis and rice, the molecular information on red algal galactosyltransferases is limited [21].

In addition to agar biosynthetic enzymes, red algae can store other unique carbohydrates, such as floridean starch and floridoside, in addition to agar and carrageenan. Additionally, cellulose, mannan, xylans, and certain unique sulfated polysaccharides constitute a large fraction of the cell wall matrix [41]. The enzymes catalyzing the synthesis and breakage of glycosidic bonds in complex sugars, multiple GH and GT families, are discussed in this paper. The differences of the GH and GT families between the aforementioned species indicated that more GH and GT families are present in the brown algae $S$. japonica than those in the two red algae C. crispus and Gp. lemaneiformis; and more GH and GT families exist in Gp. lemaneiformis than in C. crispus.

Another disaccharide trehalose may serve as a signaling molecule in plants [42]. One product of TPS, i.e., trehalose-6-phosphate, is also recognized as a regulator of sugar metabolism in plants [43] and acts as an inhibitor of SnRK1 (SNF1-related protein kinase) activity. SnRK1 has been shown to be involved in the sucrose, starch and raffinose family oligosaccharide pathways [44, 45]. There are three different pathways and total four enzymes involved in the biosynthesis and degradation of trehalose. Except for trehalose synthase, which has been reported only in a few bacteria [46], other three trehalose enzymes were all found in the Gp. lemaneiformis genome.

Phytohormones are a large category of widely distributed chemicals in plants that not only regulate plant 
growth at low concentrations but also can act as signaling molecules that participate in defense reactions. Phytohormones exist not only in higher plants but also in marine algae $[47,48]$. Although the physiological roles of phytohormones in algae are similar to those in higher plants, phytohormone biosynthetic pathways and metabolic mechanisms in algae remain elusive. In our work, we determined the contents of five phytohormones, IAA, ABA, JA, SA and cinnamic acid, in Gp. lemaneiformis using Gas Chromatography-Mass Spectrometer (GC-MS) [13]. Moreover, we found that exogenous 24-epibrassinolide could promote algal growth and agar synthesis in Gp. lemaneiformis under normal and high-temperature conditions [40]. Transcriptomic analyses showed that polar auxin transport, auxin signal transduction, and their crosstalk with other endogenous plant hormones, including zeatin, ABA, ethylene, SA, and brassinosteroids, were important in adventitious branch formation in G. lichenoides [49]. In this paper, we analyzed the components involved in phytohormone signaling pathways and identified numerous phytohormone-related genes.

\section{Conclusions}

In the present study, we performed an $88.98 \mathrm{Mb}$ draft genome assembly (approximately $91 \%$ of the entire genome size) and identified 9281 genes in the tetrasporophyte of the macroalga Gp. lemaneiformis (Rhodophyta). We then focused on carbohydrate metabolism, analyzing unique agar-synthesis enzymes and multiple GH, GT enzymes different from those of the C. crispus and $S$. japonica genomes. Finally, we summarized phytohormone signaling pathways, including those of auxin, ABA, SA and JAs, of which there is little research to date on red algae. The results will enhance our understanding of marine algal genomic information and might be helpful in the breeding and culture of this alga.

\section{Methods}

\section{Sample preparation and DNA extraction}

The tetrasporophytes of Gp. lemaneiformis strain 981 was collected at Ningde $\left(26^{\circ} 65^{\prime} \mathrm{N}, 119^{\circ} 66^{\prime} \mathrm{E}\right)$, Fujian, China, on September 20, 2009. Gp. lemaneiformis 981 is cultured in large areas of the southeastern coast of China, and we received permission to collect the samples from the farmer. We removed epiphytes and maintained a unialgal culture at the algal collection center of Ningbo University. The algal thalli were subjected to DNA extraction using an HP Plant DNA Kit (Omega Bio-Tek, USA).

\section{Genome sequencing and assembly}

The qualified genomic DNA was fragmented using the Covaris protocol, and fragments of $500 \mathrm{bp}$ were used for paired-end library preparation using a KAPA Hyper Prep Kit (Kapa/Roche) according to the manufacturer's instructions. The resulting library was sequenced on an Illumina HiSeq 2000 sequencer. The paired-end reads were used to assemble the contigs and then generate scaffolds using SOAPdenovo2 [50]. Sequences in the assembled contigs potentially from contaminating bacteria were removed by BLASTx searches against the NCBI non-redundant protein database.

\section{Transcriptome sequencing and analysis}

Total RNA was extracted using the RNeasy Plant Mini Kit (Qiagen, Germany) following the manufacturer's instructions. The RNA Library Prep Kit (NEB, USA) was used to generate the RNA-seq library, and paired-end sequencing was performed on an Illumina HiSeq 2000 sequencer. SOAPdenovo-Trans v1.03 was used to assemble the sequencing reads de novo using the default parameters [51].

\section{Gene prediction and annotation}

We used RepeatModeler (http://www.repeatmasker.org/ RepeatModeler.html) to detect the TEs in the Gp. lemaneiformis genome using the default parameters. Prior to gene prediction, the detected TEs were removed from the assembled genome.

Then, homolog-based, ab initio and transcriptome-based approaches were integrated to predict the protein-coding genes in the Gp. lemaneiformis genome according to Ye et al. [25]. Furthermore, Augustus version2.5.5 [52] and GeneMarkES version 3.0.1 [53] were utilized for ab initio gene prediction. The program Exonerate v2.2.0 [54] was used with the protein sequences of $A$. thaliana, C. merolae, $P$. tricornutum and $C$. crispus, which were downloaded from Phytozome (http://www.phytozome.net), to perform homolog-based prediction. We mapped the RNA-seq data to the genome and assembled the transcripts to the gene models using TopHat and Cufflinks [55]. The gene models predicted from the abovementioned strategies were combined into a non-redundant consensus of gene structures using EvidenceModeler [56]. Finally, for the functional annotation of the protein-coding genes, BLASTp with a cut-off E-value of 1e-10 was performed against the NCBI non-redundant protein sequence database, and those genes with the best BLAST hits were combined into the gene set. The predicted genes were mapped to the KEGG protein database for metabolic pathway analysis.

\section{Additional files}

Additional file 1: Table S1. Comparison of the number of enzymes involved in agar synthesis in the 6 species. (DOCX $26 \mathrm{~kb}$ )

Additional file 2: Table S2. The enzymes involved in converting the agar precursor into agar in Gp. lemaneiformis. (DOCX 24 kb) 
Additional file 3: Table S3. The numbers of glycoside hydrolases (GHs) identified in the Gp. lemaneiformis, C. crispus and S. japonica genomes. (DOCX $26 \mathrm{~kb})$

Additional file 4: Table S4. The numbers of glycosyltransferases (GTs) identified in the Gp. lemaneiformis, C. crispus and S. japonica genomes. (DOCX $26 \mathrm{~kb}$ )

Additional file 5: Table S5. The enzymes involved in trehalose metabolism. (DOCX $24 \mathrm{~kb}$ )

Additional file 6: Figure S1. The phylogenetic relationship of cellulose synthase (CESA) in Gp. lemaneiformis with those of other related species. The tree was constructed based on the maximum likelihood method with 1000 bootstrap replicates using Mega5.10 software. (DOCX 27 kb)

Additional file 7: Table S6. The genes related to auxin signaling in Gp. lemaneiformis. (DOCX $24 \mathrm{~kb}$ )

Additional file 8: Table S7. The genes related to abscisic acid signaling in Gp. lemaneiformis. (DOCX $24 \mathrm{~kb}$ )

Additional file 9: Table S8. The enzymes related to salicylic acid signaling in Gp. lemaneiformis. (DOCX $25 \mathrm{~kb}$ )

Additional file 10: Table S9. The enzymes related to jasmonic acid signaling in Gp. lemaneiformis. (DOCX $24 \mathrm{~kb}$ )

Additional file 11: Figure S2. Phylogeny deduced from the lipoxygenases (LOX) of ten species. The tree was constructed based on the maximum likelihood method with 1000 bootstrap replicates using Mega5.10 software. (DOCX $24 \mathrm{~kb})$

\section{Abbreviations}

ABA: Abscisic acid; AOC: Allene oxide cyclase; AOS: Allene oxide synthase BP: Biological process; CC: Cellular component; CESA: Cellulose synthase; CSL: Cellulose synthase-like; GC-MS: Gas chromatography-mass spectrometer; GHs: Glycoside hydrolases; GO: Gene ontology; GTs: Glycosyltransferases; IAA: Indole-3-acetic acid; ICS: Isochorismate synthase; IPL: Isochorismate pyruvate lyase; JA: Jasmonic acid; JAs: Jasmonates; KEGG: Kyoto encyclopedia of genes and genomes; LINEs: Long interspersed repeated DNA elements; LOX: Lipoxygenase; LTR: Long terminal repeat; MF: Molecular function; MTs: Methyltransferases; OPR3: 12-oxophytodienoate reductase 3; PAL: Phenylalanine ammonia lyase; PET: Paired-end-tag; PTs: Pyruvyl transferases; RC/Helitron: Rolling-circle transposons; SA: Salicylic acid; SHs: Sulfohydrolases; SnRK: SNF1-related protein kinase; STs: Sulfotransferases; TEs: Transposable elements; TPS: Trehalose-phosphate synthase

\section{Funding}

This research was funded by the National Natural Science Foundation of China (41376151, 31672674). This work was also supported by the Key Program of the Natural Science Foundation of Zhejiang Province (Z17D060001), the Ningbo International Cooperation Program of Science and Technology (2017D10019), and the K. C. Wong Magna Fund of Ningbo University. The funding agencies did not play a role in the experimental design, results analysis or writing of the manuscript but did provide financial support for the manuscript.

\section{Availability of data and materials}

The whole-genome shotgun project and the gene assembly information have been submitted to and deposited in the GenBank database under BioProject ID SRP106236.

\section{Authors' contributions}

$X S, N X$ and $X Z$ developed the concept and interpreted the results. FW performed the genomic DNA and RNA isolation. TS and YK were responsible for sequencing. SOH and JW performed the genome assembly, genome annotation and data analysis. XS drafted the manuscript. RY and GW reviewed the manuscript and provided valuable feedback. All authors have read and approved the final version of this manuscript.

\section{Ethics approval and consent to participate}

Not applicable.

\section{Competing interests}

The authors declare that they have no competing interests.

\section{Publisher's Note}

Springer Nature remains neutral with regard to jurisdictional claims in published maps and institutional affiliations.

\section{Author details}

${ }^{1}$ Key Laboratory of Marine Biotechnology of Zhejiang Province, School of Marine Sciences, Ningbo University, Ningbo 315211, People's Republic of China. ${ }^{2}$ School of Biomedical Engineering, Shanghai Center for Systems Biomedicine, Shanghai Jiao Tong University, Shanghai 200240, People's Republic of China. ${ }^{3}$ Institute of Oceanology, Chinese Academy of Sciences, Qingdao 266071, People's Republic of China. ${ }^{4}$ Department of Biomedicine, Aarhus University, 8000 Aarhus C, Denmark.

Received: 14 August 2017 Accepted: 10 May 2018

Published online: 25 May 2018

\section{References}

1. Qiu H, Price DC, Yang EC, Yoon HS, Bhattacharya D. Evidence of ancient genome reduction in red algae (Rhodophyta). J Phycol. 2015;51:624-36.

2. Yoon HS, Hackett JD, Ciniglia C, Pinto G, Bhattacharya D. A molecular timeline for the origin of photosynthetic eukaryotes. Mol Biol Evol. 2004;21: 809-18.

3. Bird CJ, Ragan MA, Critchley AT, Rice EL, Gutell RR. Molecular relationships among the Gracilariaceae (Rhodophyta): further observations on some undetermined species. Eur J Phycol. 1994;29:195-202.

4. Gurgel CFD, Liao LM, Frederica S, Hommersand MH. Systematics of Gracilariopsis Dawson (Gracilariales, Rhodophyta) based on rbcL sequence analysis and morphological evidence. J Phycol. 2003;39:154-71.

5. Yu J, Yang YF. Physiological and biochemical response of seaweed Gracilaria lemaneiformis to concentration changes of $\mathrm{N}$ and P. J Exp Mar Biol Ecol. 2008;367:142-8

6. Zhou Y, Yang H, Hu H, Liu Y, Mao Y, Zhou H, et al. Bioremediation potential of the macroalga Gracilaria lemaneiformis (Rhodophyta) integrated into fed fish culture in coastal waters of North China. Aquaculture. 2006;252:264-76.

7. Yang Y, Liu Q, Chai ZY, Tang Y. Inhibition of marine coastal bloom-forming phytoplankton by commercially cultivated Gracilaria lemaneiformis (Rhodophyta). J Appl Phycol. 2015;27:1-12.

8. Liao X, Yang L, Chen M, Yu J, Zhang S, Ju Y. The hypoglycemic effect of a polysaccharide (GLP) from Gracilaria lemaneiformis and its degradation products in diabetic mice. Food Funct. 2015;6:2542-9.

9. Fan $\mathrm{YL}$, Wang $\mathrm{WH}$, Chen HS, Liu N, Liu AJ. In vitro and in vivo immunomodulatory activities of an acidic polysaccharide from Gracilaria lemaneiformis. Adv Mater Res. 2012;468-471:1941-5.

10. Kang Y, Wang Z, Xie D, Sun X, Yang W, Zhao X, et al. Characterization and antitumor activity of polysaccharide from Gracilariopsis lemaneiformis. Marine Drugs. 2017;15:100.

11. Bixler $\mathrm{HJ}$, Porse $\mathrm{H}$. A decade of change in the seaweed hydrocolloides industry. J Appl Phycol. 2011;23:321-35.

12. González-Leija JA, Hernández-Garibay E, Pacheco-Ruíz I, Guardado-Puentes J, Espinoza-Avalos J, López-Vivas JM, et al. Optimization of the yield and quality of agar from Gracilariopsis lemaneiformis (Gracilariales) from the Gulf of California using an alkaline treatment. J Appl Phycol. 2009;21:321-6.

13. Lu Y, Xu J. Phytohormones in microalgae: a new opportunity for microalgal biotechnology? Trends Plant Sci. 2015;20:273-82

14. Cai X, Shao M, Sun X, Xu N. Detection of multiple phytohormones by GCMS technique in Gracilaria lemaneiformis and the response to nitrogen stresses. Oceanol Limnol Sin. 2011:42:753-8. (Chinese with English abstract)

15. Zhang L, Wang X, Qian H, Chi S, Liu C, Liu T. Complete sequences of the mitochondrial DNA of the wild Gracilariopsis lemaneiformis and two mutagenic cultivated breeds (Gracilariaceae, Rhodophyta). PLoS One. 2012;7:e40241.

16. Du Q, Bi G, Mao Y, Sui Z. The complete chloroplast genome of Gracilariopsis lemaneiformis (Rhodophyta) gives new insight into the evolution of family Gracilariaceae. J Phycol. 2016;52:441-50.

17. Zhou W, Hu Y, Sui Z, Fu F, Wang J, Chang L, et al. Genome survey sequencing and genetic background characterization of Gracilariopsis lemaneiformis (Rhodophyta) based on next-generation sequencing. PLoS One. 2013:8:e69909.

18. Kapraun DF, Dutcher JA. Cytophotometric estimation of inter and intraspecific nuclear DNA content variation in Gracilaria and Gracilariopsis (Gracilariales, Rhodophyta). Bot Mar. 1991;34:139-44. 
19. Kapitonov W, Jurka J. Helitrons on a roll: eukaryotic rolling-circle transposons. Trends Genet. 2007;23:521-9.

20. Chang L, Sui Z, Fu F, Zhou W, Wang J, Kang KH, et al. Relationship between gene expression of UDP-glucose pyrophosphorylase and agar yield in Gracilariopsis lemaneiformis (Rhodophyta). J Appl Phycol. 2014;26:2435-41.

21. Lee WK, Lim YY, Leow ATC, Namasivayam P, Abdullah JO, Ho CL. Biosynthesis of agar in red seaweeds: a review. Carbohydr Polym. 2017; 164:23-30.

22. Negishi M, Pedersen LG, Petrotchenko E, Shevtsov S, Gorokhov A, Kakuta Y, et al. Structure and function of sulfotransferases. Arch Biochem Biophys. 2001:390:149-57.

23. Collén J, Porcel B, Carré W, Ball SG, Chaparro C, Tonon T, et al. Genome structure and metabolic features in the red seaweed Chondrus crispus shed light on evolution of the Archaeplastida. Proc Natl Acad Sci U S A. 2013;110: 5247-52.

24. Michel G, Tonon T, Scornet D, Cock JM, Kloareg B. The cell wall polysaccharide metabolism of the brown alga Ectocarpus siliculosus. Insights into the evolution of extracellular matrix polysaccharides in eukaryotes. New Phytol. 2010;188:82-97.

25. Ye N, Zhang X, Miao M, Fan X, Zheng Y, Xu D, et al. Saccharina genomes provide novel insight into kelp biology. Nat Commun. 2015;6:6986.

26. Yin Y, Huang J, Xu Y. The cellulose synthase superfamily in fully sequenced plants and algae. BMC Plant Biol. 2009;9:99.

27. Roberts $E$, Roberts AW. A cellulose synthase (CESA) gene from the red alga Porphyra yezoensis (Rhodophyta). J Phycol. 2009;45:203-12.

28. Matthews PR, Schindler M, Howles P, Arioli T, Williamson RE. A CESA from Griffithsia monilis (Rhodophyta, Florideophyceae) has a family 48 carbohydrate-binding module. J Exp Bot. 2010;61:4461-8.

29. Nobles DR, Brown RM. The pivotal role of cyanobacteria in the evolution of cellulose synthases and cellulose synthase-like proteins. Cellulose. 2004;11:437-48.

30. Yokoya NS, Stirk WA, van Staden J, Novák O, Turečková V, Pénćík A, et al Endogenous cytokinins, auxins, and abscisic acid in red algae from Brazil. $J$ Phycol. 2010;46:1198-205.

31. Huizinga DH, Denton R, Koehler KG, Tomasello A, Wood L, Sen SE, et al. Farnesylcysteine lyase is involved in negative regulation of abscisic acid signaling in Arabidopsis. Mol Plant. 2010;3:143-55.

32. Chen Z, Zheng Z, Huang J, Lai Z, Fan B. Biosynthesis of salicylic acid in plants. Plant Signal Behav. 2009:4:493-6.

33. Wasternack C, Hause B. Jasmonates: biosynthesis, perception, signal transduction and action in plant stress response, growth and development. An update to the 2007 review in annals of botany. Ann Bot. 2013;111:1021-58.

34. Bannenberg G, Martínez M, Hamberg M, Castresana C. Diversity of the enzymatic activity in the lipoxygenase gene family of Arabidopsis thaliana. Lipids. 2009;44:85-95.

35. Stenzel I, Hause B, Miersch O, Kurz T, Maucher H, Weichert $\mathrm{H}$, et al. Jasmonate biosynthesis and the allene oxide cyclase family of Arabidopsis thaliana. Plant Mol Biol. 2003:51:895-911.

36. Bhattacharya D, Price DC, Chan CX, Qiu H, Rose N, Ball S, et al. Genome of the red alga Porphyridium purpureum. Nat Commun. 2013:4:1941.

37. Radakovits R, Jinkerson RE, Fuerstenberg SI, Tae H, Settlage RE, Boore JL, et al. Draft genome sequence and genetic transformation of the oleaginous alga Nannochloropsis gaditana. Nat Commun. 2012;3:686

38. Siow RS, Teo SS, Ho WY, Shukor MYA, Phang SM, Ho CL. Molecular cloning and biochemical characterization of galactose-1-phosphate uridylyltransferase from Gracilaria changii (Rhodophyta). J Phycol. 2012;48:155-62.

39. Li M, Sui Z, Kang K, Zhang X, Zhu M, Yan B. Cloning and analysis of the galactose-1-phosphate uridylyltransferase (galt) gene of Gracilariopsis lemaneiformis (Rhodophyta) and correlation between gene expression and agar synthesis. J Appl Phycol. 2010;22:157-64.

40. Tang $X, X u$ N, Sun $X$, Li Y, Zhang L. Effects of 24-epibrassinolide on the agar synthesis and expression of genes involved in marine alga Gracilariopsis lemaneiformis. J Fish China. 2015:39:1788-98. (in Chinese with English abstract)

41. Popper ZA, Michel G, Hervé C, Domozych DS, Willats WG, Tuohy MG, et al. Evolution and diversity of plant cell walls: from algae to flowering plants. Annu Rev Plant Biol. 2011;62:567-90.

42. Elbein AD, Pan YT, Pastuszak I, Carroll D. New insights on trehalose: a multifunctional molecule. Glycobiology. 2003;13:17R-27R.

43. Zhang Y, Primavesi LF, Jhurreea D, Andralojc PJ, Mitchell RAC, Powers SJ, et al. Inhibition of SNF1-related protein kinase1 activity and regulation of metabolic pathways by trehalose-6-phosphate. Plant Physiol. 2009;149:1860-71.
44. Wang C, Zhang Z, Miao M. SNF1-related protein kinase (SnRK) 1 involved in the regulation of raffinose family oligosaccharide metabolism in cucumber (Cucumis sativus L.) calli. J Plant Growth Regul. 2016;35:851-64.

45. Paul MJ, Primavesi LF, Jhurreea D, Zhang Y. Trehalose metabolism and signaling. Ann Rev Plant Biol. 2008;59:417-41.

46. Avonce N, Mendoza-Vargas A, Morett E, Iturriaga G. Insights on the evolution of trehalose biosynthesis. BMC Evol Biol. 2006;6:109-23.

47. Stirk WA, Novák O, Hradecká V, Pěnčík A, Rolčík J, Strnad M, et al. Endogenous cytokinins, auxins and abscisic acid in Ulva fasciata (Chlorophyta) and Dictyota humifusa (Phaeophyta): towards understanding their biosynthesis and homoeostasis. Eur J Phycol. 2009:44:231-40.

48. Tarakhovskaya ER, Maslov YI, Shishova MF. Phytohormones in algae. Russ J Plant Physiol. 2007;54:163-70

49. Wang W, Li H, Lin X, Yang S, Wang Z, Fang B. Transcriptome analysis identifies genes involved in adventitious branches formation of Gracilaria lichenoides in vitro. Sci Rep. 2015;5:17099.

50. Luo R, Liu B, Xie Y, Li Z, Huang W, Yuan J, et al. SOAPdenovo2: an empirically improved memory-efficient short-read de novo assembler. Gigascience. 2012;1:18.

51. Xie Y, Wu G, Tang J, Luo R, Patterson J, Liu S, et al. SOAPdenovo-trans: de novo transcriptome assembly with short RNA-Seq reads. Bioinformatics. 2014:30:1660-6.

52. Stanke M, Schöffmann O, Morgenstern B, Waack S. Gene prediction in eukaryotes with a generalized hidden Markov model that uses hints from external sources. BMC Bioinformatics. 2006;7:62.

53. Ter-Hovhannisyan V, Lomsadze A, Chernoff YO, Borodovsky M. Gene prediction in novel fungal genomes using an ab initio algorithm with unsupervised training. Genome Res. 2008;18:1979-90.

54. Slater GS, Birney E. Automated generation of heuristics for biological sequence comparison. BMC Bioinformatics. 2005;6:31.

55. Trapnell C, Roberts A, Goff L, Pertea G, Kim D, Kelly DR, et al. Differential gene and transcript expression analysis of RNA-seq experiments with TopHat and cufflinks. Nat Protoc. 2012;7:562-78.

56. Hass BJ, Salzberg SL, Zhu W, Pertea M, Allen JE, Orvis J, et al. Automated eukaryotic gene structure annotation using EVidenceModeler and the program to assemble spliced alignments. Genome Biol. 2008;9:R7.

\section{Ready to submit your research? Choose BMC and benefit from:}

- fast, convenient online submission

- thorough peer review by experienced researchers in your field

- rapid publication on acceptance

- support for research data, including large and complex data types

- gold Open Access which fosters wider collaboration and increased citations

- maximum visibility for your research: over $100 \mathrm{M}$ website views per year

At BMC, research is always in progress.

Learn more biomedcentral.com/submissions 